\title{
Transverse impedance measurement in RHIC and the AGS
}

\author{
N. Biancacci, M. Blaskiewicz, Y. Dutheil, \\ C. Liu, K. Mernick, M. Minty, S.M. White
}

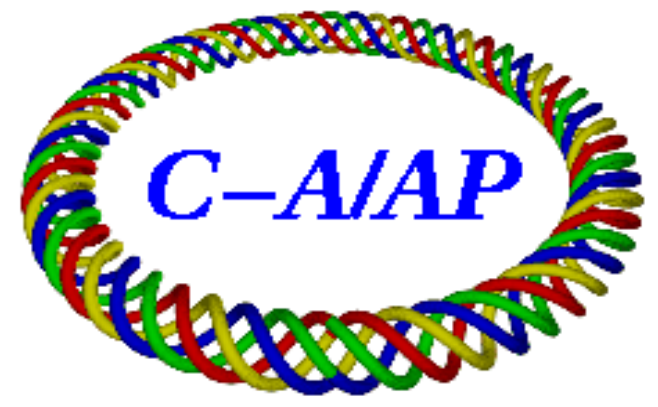

\section{Collider-Accelerator Department Brookhaven National Laboratory Upton, NY 11973}

Notice: This document has been authorized by employees of Brookhaven Science Associates, LLC under Contract No. DE-AC02-98CH10886 with the U.S. Department of Energy. The United States Government retains a nonexclusive, paid-up, irrevocable, world-wide license to publish or reproduce the published form of this document, or allow others to do so, for United States Government purposes. 


\title{
Transverse Impedance Measurement in RHIC and the AGS
}

Nicolo Biancacci (CERN), M. Blaskiewicz, Y. Dutheil, C. Liu, K. Mernick, M. Minty, S.M. White (BNL)

May 12, 2014

\begin{abstract}
The RHIC luminosity upgrade program aims for an increase of the polarized proton luminosity by a factor 2. To achieve this goal a significant increase in the beam intensity is foreseen. The beam coupling impedance could therefore represent a source of detrimental effects for beam quality and stability at high bunch intensities. For this reason it is essential to quantify the accelerator impedance budget and the major impedance sources, and possibly cure them.

In this MD note we summarize the results of the 2013 transverse impedance measurements in the AGS and RHIC. The studies have been performed measuring the tune shift as a function of bunch intensity and deriving the total accelerator machine transverse impedance. For RHIC, we could obtain first promising results of impedance localization measurements as well.
\end{abstract}




\section{Introduction}

The RHIC luminosity upgrade program aims for an increase of the polarized proton luminosity by a factor 2 . To achieve this goal a significant increase in the beam intensity is foreseen. The beam coupling impedance could therefore represent a source of detrimental effects for beam quality and stability at high bunch intensities. For this reason it is essential to quantify the accelerator impedance budget and the major impedance sources, and possibly cure them.

In this MD note we resume the results of te 2013 transverse impedance measurements in the AGS and RHIC. Past measurements can be found in 11. The studies have been performed measuring the tune shift as a function of bunch intensity and deriving the total accelerator machine. For RHIC, we could obtain first promising results of impedance localization measurements as well.

In the first section we will present the results of the vertical impedance measurement in the AGS, in the second section we will present tune shift measurements in RHIC and in the third the first attempts of impedance localization through the turn by turn RHIC BPM system.

\subsection{AGS measurements}

In the AGS accelerator the absence of a multi-turn BPM system allowed only for the measurement of the total effective impedance through the observation of the transverse tune shift with intensity. A measurement in the vertical plane could be performed while time constraints didn't allow for the horizontal one (which is anyway believed to be small due to the vacuum chamber elliptical shape).

The measurement has been performed at flat top before extraction with the machine parameters listed in App. A. The used beam parameters are reported in Tab. 1. Figure 1 shows the beam profile measured at the bunch shape monitor (BSM) and the rms bunch length $\sigma_{b}$ with a Gaussian fit is $\sigma_{b}=(5.8 \pm 0.1) \mathrm{ns}$, over 25 measurements.

\begin{tabular}{lcr}
\hline \hline AGS beam & Parameter & Value \\
\hline Intensity & $N_{b}$ & $5 \cdot 10^{12} \rightarrow 25 \cdot 10^{12} \mathrm{ppb}$ \\
Momentum spread & $\mathrm{d} p / p_{o}$ & $2 \cdot 10^{-4}$ \\
Bunch length & $\sigma_{t}$ & $5.8 \mathrm{~ns}$ \\
Normalized Horizontal emittance & $\varepsilon_{x_{n}}$ & $13.5 \mu \mathrm{rad}$ \\
Normalized Vertical emittance & $\varepsilon_{y_{n}}$ & $13.5 \mu \mathrm{rad}$ \\
\hline
\end{tabular}

Table 1: AGS beam parameters.

An intensity scan was set up from $5 \cdot 10^{12}$ up to $25 \cdot 10^{12} \mathrm{ppb}$ and the beam was excited by a tune kicker synchronized with a dedicated high-resolution BPM from which 300 turns of coherent betatron oscillation were stored. The acquired tune data are shown in Fig. 2. a tune shift of $5 \cdot 10^{-5}$ per $10^{11}$ can be measured. To obtain the total effective transverse impedance $Z_{y}^{\text {eff }}$ defined as

$$
Z_{y}^{e f f}=\frac{\int_{-\infty}^{+\infty} Z_{y}(\omega) S(\omega) \mathrm{d} \omega}{\int_{-\infty}^{+\infty} S(\omega) \mathrm{d} \omega},
$$

with $S(\omega)$ the beam spectrum and $Z_{y}(\omega)$ the vertical impedance, we can use Eq. (2) 2

$$
\Delta Q_{y}=-\frac{q^{2} N_{b} R}{8 \pi^{3 / 2} Q_{y o} \beta^{2} E_{o} \sigma_{\tau}} \operatorname{Im}\left(Z_{y}^{\text {eff }}\right),
$$

where $Q_{y}$ is the measured vertical tune (i.e. perturbed by the impedance), $Q_{y o}$ the unperturbed vertical tune (i.e. extrapolated at 0 -current), $q$ the proton charge, $N_{b}$ the bunch population, $R$ 


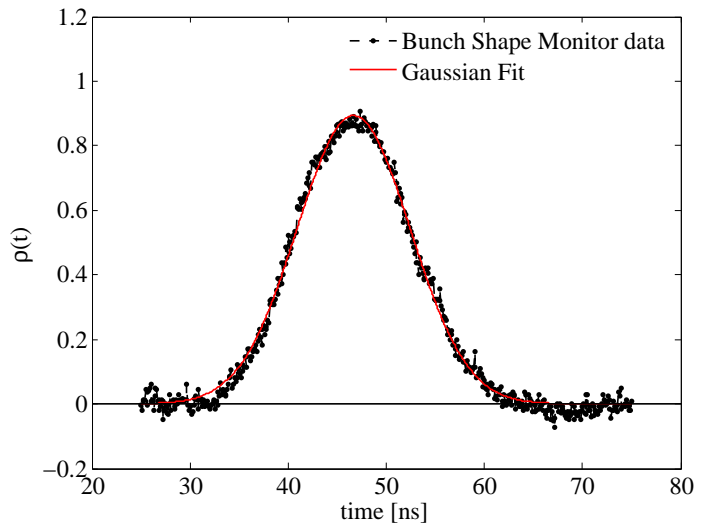

(a)

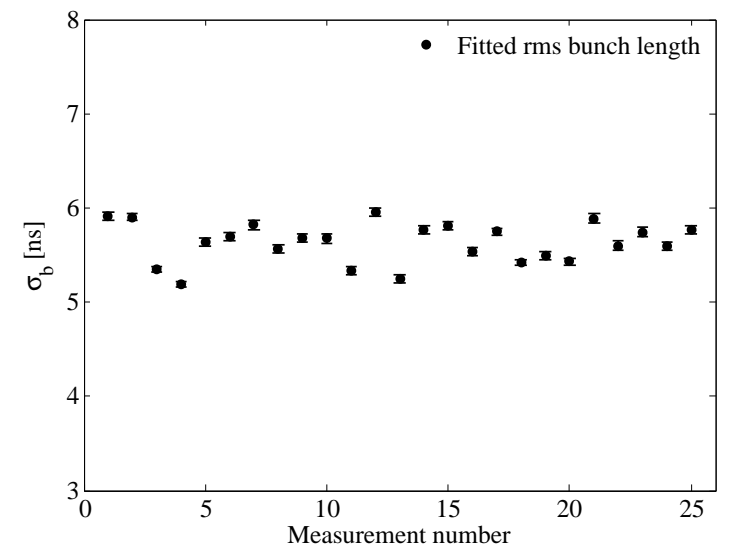

(b)

Figure 1: Example of acquired bunch shape (a) and rms bunch length from Gaussian fit from 25 measurements (b) in the AGS.

the machine radius, $E_{o}$ the proton total energy, $\sigma_{\tau}$ the bunch length (in seconds). Inverting the relation and taking the derivative over the intensity scan, we obtain

$$
Z_{y}^{e f f}=1.3 \pm 0.1 \mathrm{M} \Omega / \mathrm{m}
$$

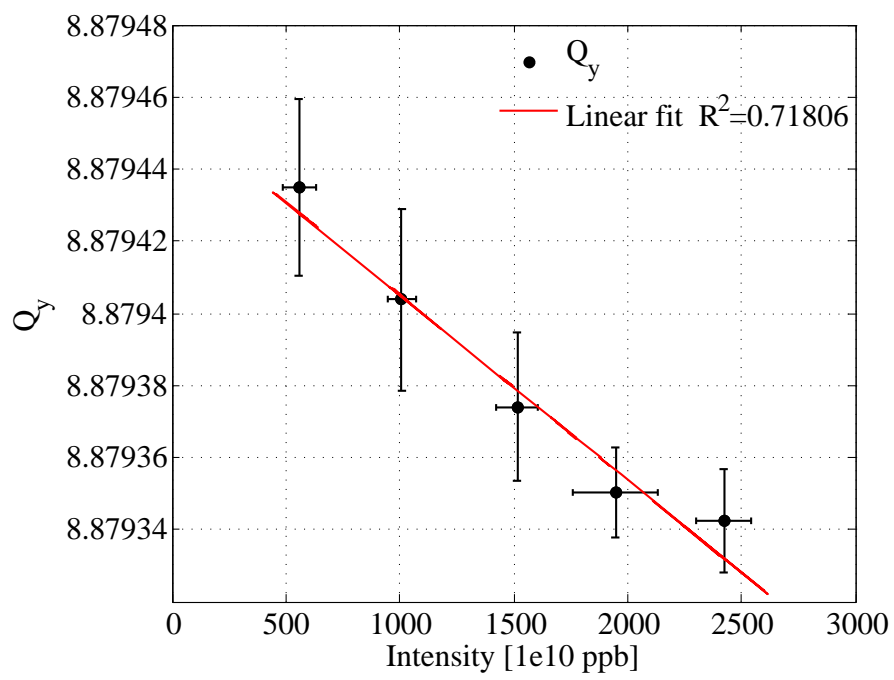

Figure 2: Tune shift with intensity acquired in the AGS vertical plane.

From past measurements $[3]$ it has been considered a longitudinal effective impedance $Z_{l}^{\text {eff }} / n=10 \Omega$ where we recall the longitudinal effective impedance definition

$$
\frac{Z_{l}^{\text {eff }}}{n}=\frac{\int_{-\infty}^{\infty} \frac{Z_{l}(\omega)}{n} S(\omega) \mathrm{d} \omega}{\int_{-\infty}^{\infty} S(\omega) \mathrm{d} \omega},
$$

with $n=\omega / \omega_{o}$ and $\omega_{o}=2 \pi f_{\text {rev }}$. Assuming the longitudinal impedance as prevalently due to the resistive wall, we can derive the transverse effective impedance comparing their analytical expressions [4] obtaining

$$
Z_{t}(\omega)=\frac{\beta c}{\omega} \frac{2}{b^{2}} Z_{l}(\omega)
$$


where $b$ is the beam pipe radius. Since in most of the AGS machine the vacuum chamber is elliptical with minor axis $8.2 \mathrm{~cm}$ and major axis $17 \mathrm{~cm}$ long, we assume $b$ as the minor semi axis length, i.e. $4.1 \mathrm{~cm}$. Integrating over the beam spectrum $S(\omega)$ we get

$$
Z_{t}^{e f f}=\frac{2 R}{b^{2}} \frac{Z_{l}^{e f f}}{n} \simeq 1.5 \mathrm{M} \Omega / \mathrm{m}
$$

which is close to the measured one. This implies that most of the AGS impedance could be considered due to the resistive wall contribution. In order to give further confirmation to this result, the measurement of the effective longitudinal impedance could be updated unless not significant machine changes are expected to be occurred since 2003.

\section{RHIC measurements}

The impedance measurements in RHIC were performed on 24-04-2013, 1-05-2013 and 15-05-2013 in collaboration with the machine operation team. Both total and local machine impedance could be in principle measured thanks to the high-performance of the BPM system. Focusing on the phase advance measurement accuracy for the impedance localization, we consider Eq. (7) [5],

$$
\sigma_{\Delta \mu / \Delta N_{b}}=F_{\Delta \mu} \frac{N S R}{\sigma_{X} \sqrt{M} \sqrt{N}},
$$

where the involved parameters are reported in Tab. 2

\begin{tabular}{lcr}
\hline \hline Best BPM performance & Parameter & Value \\
\hline Maximum number of turns & $N$ & 1000 \\
Noise level & $\sigma_{n}$ & $15 \mu \mathrm{m}$ \\
Kick amplitude & $A_{k i c k}$ & $2 \mathrm{~mm}$ \\
Number of measurements & $M$ & 100 \\
Intensity scan & $X$ & $0.5 \cdot 10^{11} \rightarrow 2 \cdot 10^{11} \mathrm{ppb}$ \\
Phase advance slope accuracy & $\sigma_{\Delta \mu / \Delta N_{b}}$ & $6 \cdot 10^{-5} 10^{-11} \mathrm{rad} / 2 \pi$ \\
\hline
\end{tabular}

Table 2: Phase advance slope accuracy estimation for the RHIC BPM system.

In order to assess the feasibility of the localization measurement, he phase advance slope accuracy $\sigma_{\Delta \mu / \Delta N_{b}}$ can be compared with the whole accelerator tune shift $\Delta Q_{y} / \Delta N_{b}$.

\subsubsection{Total impedance measurements}

The total accelerator impedance could be derived from the tune shift with intensity for both accelerators, Blue and Yellow.

The measurements for Blue where performed on 24-04-2013 and 15-05-2013. The measurements were done injecting a train of bunches at different intensities, from $0.5 \cdot 10^{11} \mathrm{ppb}$ to $1.8 \cdot 10^{11} \mathrm{ppb}$ with average rms bunch length $\sigma_{b} \simeq 5 \mathrm{~ns}$. The beam was excited with a transverse kick in both planes 20 turns after injection, and the betatron oscillations were recorded for 1024 turns. Chromaticity was set as close as possible to 0 on both planes, requiring a long machine set-up time. An example of betatron signal from a BPM is shown in Fig. 3 were we can see a fast damping after 100 turns and coherent oscillations up to 1024 turns. The signals are analyzed with SUSSIX [6] after an SVD noise cleaning and the tune shift with intensity is therefore computed.

The tune shifts for both X an Y plane are shown in Fig. 4.

Analogously, on 01-05-2013, the same measurement was done in the Yellow ring injecting train of bunches at different intensities, from $0.5 \cdot 10^{11}$ to $2.5 \cdot 10^{11} \mathrm{ppb}$ with average rms bunch length $\sigma_{b} \simeq 4 \mathrm{~ns}$. The analyzed tune shifts are presented in Fig. 5 for a set of 2 good fills in Yellow. 


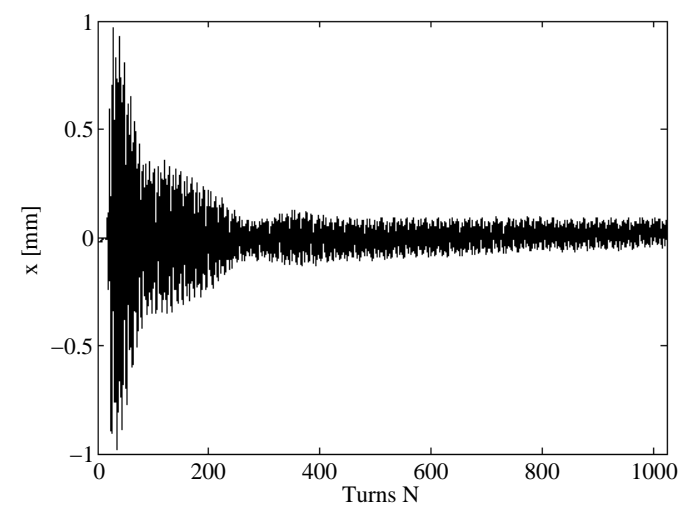

(a)

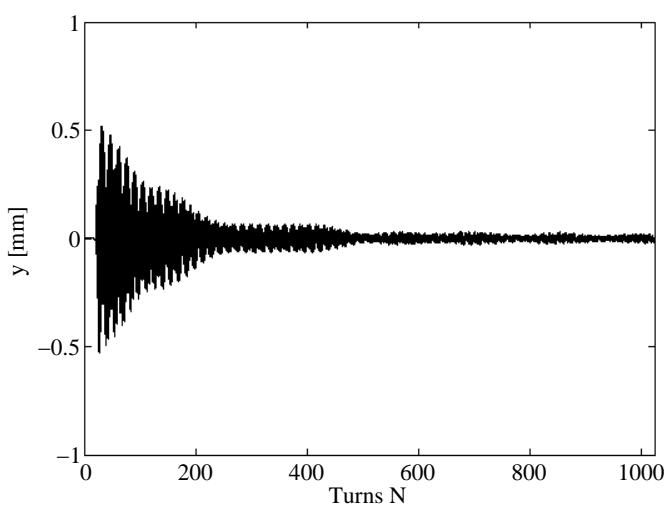

(b)

Figure 3: Example of recorded traces from 15-05-2013 from the "B06-BV15" BPM for X (a) and Y plane (b).

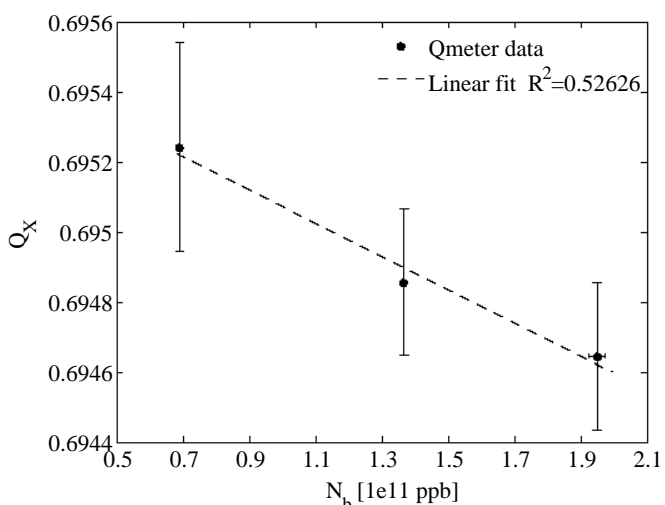

(a)

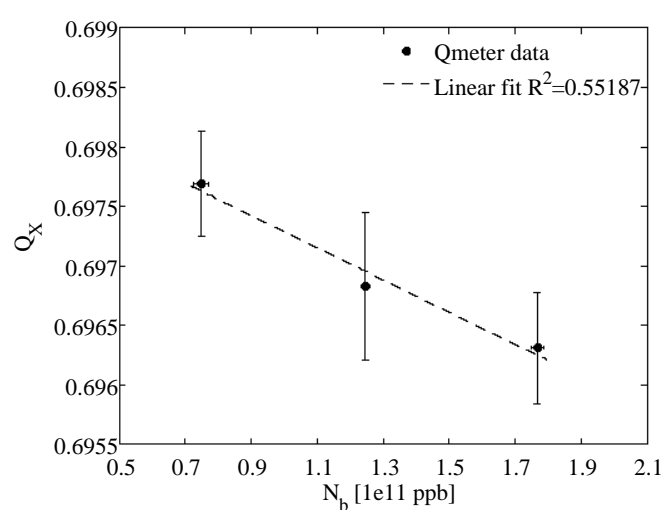

(c)

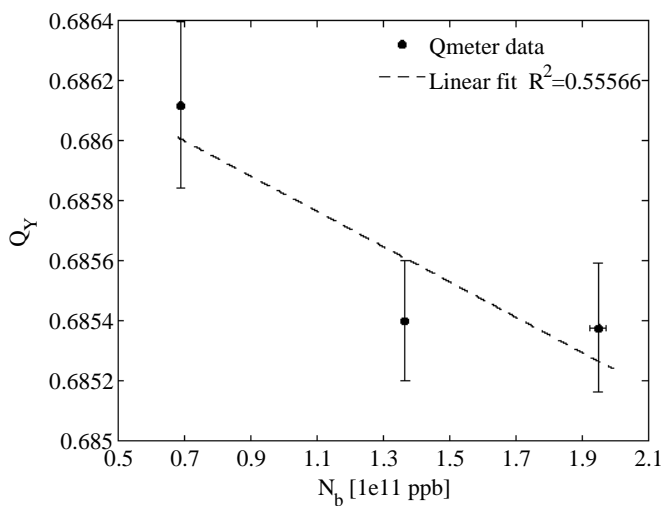

(b)

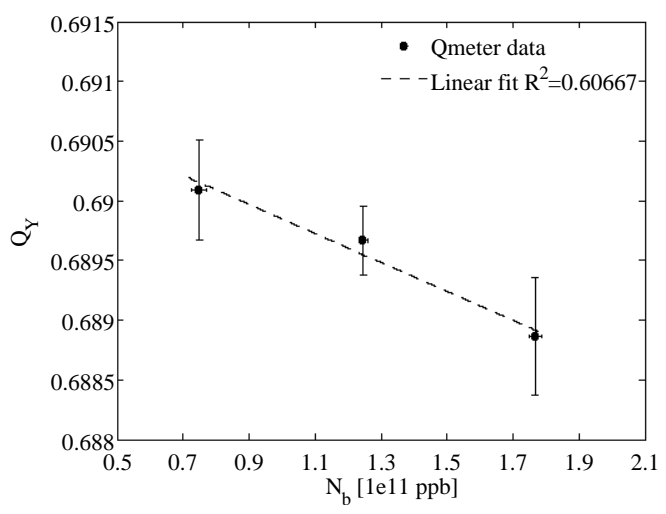

(d)

Figure 4: Tune shift measurements in RHIC Blue ring from 24-04-2013 X plane (a) and Y plane (b), 15-05-2013 X plane (c) and Y plane (d) with a bunch length $\sigma_{b} \simeq 5 \mathrm{~ns}$.

The deduced effective impedance is reported in Tabs. 3 and 4 and is calculated using Eq. (2) accounting for the average $\beta$ function along the accelerator.

We might notice that, for the tune shift measurement, the drift of the machine with time has been taken into account: at injection, the exponential decay for the current in the magnets affects the machine parameters as depicted in Fig. 66resulting in wrong tune shift estimations (up to 10\%). 


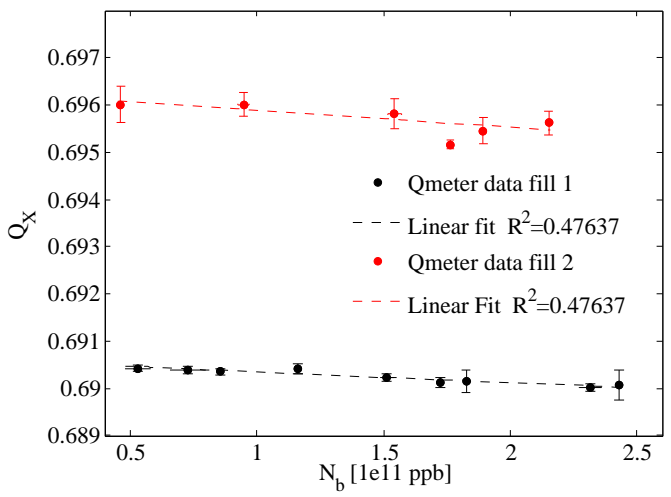

(a)

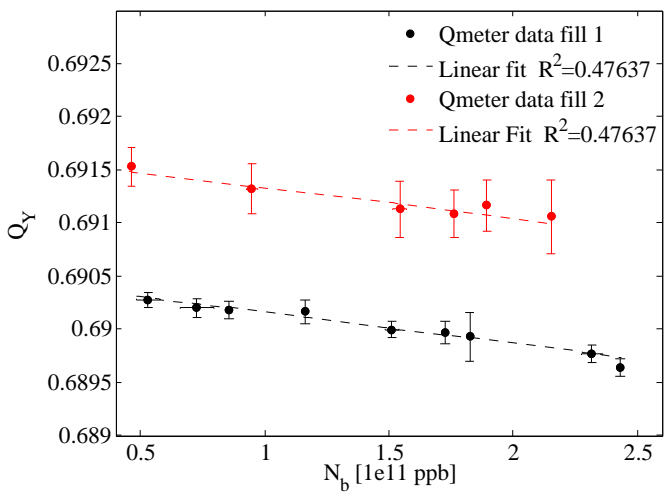

(b)

Figure 5: Tune shift measurements of two injection fills in the RHIC Yellow ring taken on 01-05$2013 \mathrm{X}$ plane (a) and Y plane (b) with a bunch length $\sigma_{b} \simeq 4 \mathrm{~ns}$.

\begin{tabular}{|c|c|c|c|c|}
\hline \hline Measurement & $Z_{x}^{\text {eff }}[\mathrm{M} \Omega / \mathrm{m}]$ & $Z_{y}^{\text {eff }}[\mathrm{M} \Omega / \mathrm{m}]$ & $\mathrm{d} Q_{x} / \mathrm{d} N_{b}\left(\cdot 10^{-11}\right)$ & $\mathrm{d} Q_{y} / \mathrm{d} N_{b}\left(\cdot 10^{-11}\right)$ \\
\hline $24-04-2013$ & $(7.06 \pm 1.29)$ & $(9.06 \pm 1.56)$ & $(-4.74 \pm 0.86) \cdot 10^{-4}$ & $(-5.88 \pm 1.01) \cdot 10^{-4}$ \\
$15-05-2013$ & $(20.51 \pm 1.98)$ & $(19.07 \pm 1.15)$ & $(-13.53 \pm 1.30) \cdot 10^{-4}$ & $(-12.16 \pm 0.73) \cdot 10^{-4}$ \\
\hline Average & $(13.79 \pm 6.72)$ & $(14.01 \pm 5.00)$ & $(-9.13 \pm 4.39) \cdot 10^{-4}$ & $(-9.02 \pm 3.14) \cdot 10^{-4}$ \\
\hline
\end{tabular}

Table 3: Tune slope and effective impedances measured in RHIC Blue ring.
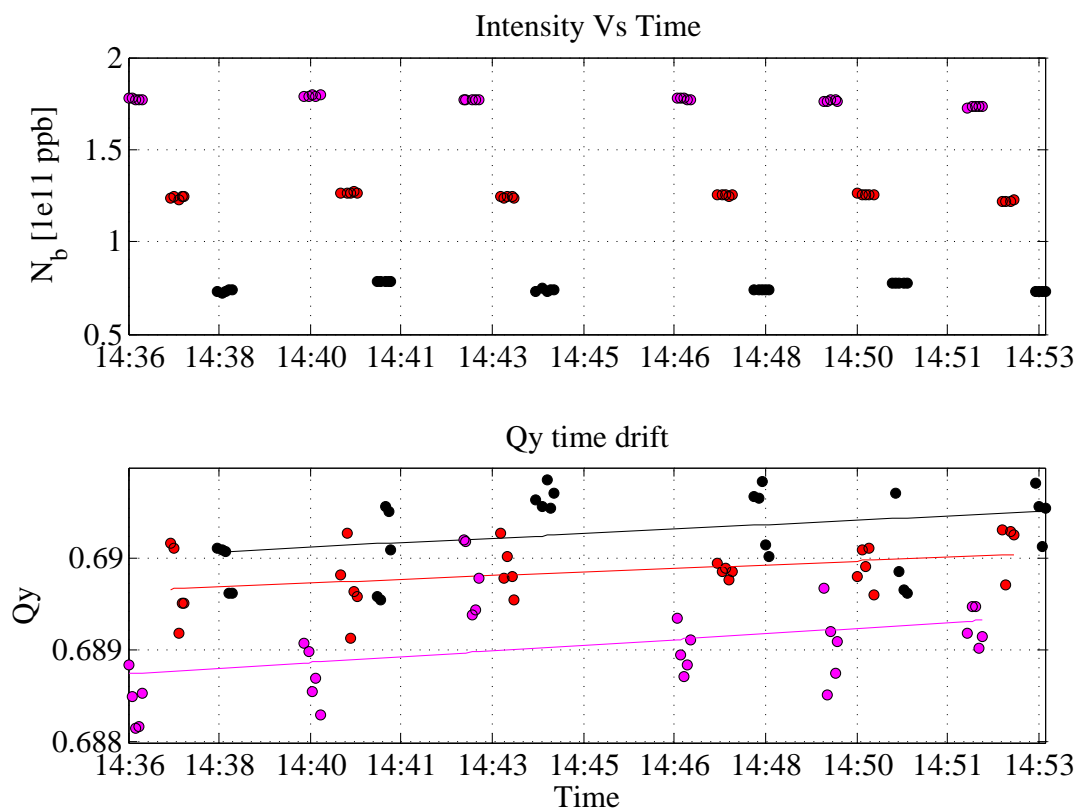

Figure 6: Example of tune drift with time for the vertical tune measured on 15-05-2013 on Blue. On top the train of injected bunches from high to low intensities; on bottom the correspondent measured tunes (dots) and the linear drift with time (thick lines).

\subsubsection{Localized impedance measurements}

The RHIC BPM system [7], 8] consists in 160 23-cm cryogenic striplines per plane per ring. Most of the devices are made of by two opposite striplines oriented vertically or horizontally accordingly 


\begin{tabular}{|c|c|c|c|c|}
\hline \hline Measurement & $Z_{x}^{\text {eff }}[\mathrm{M} \Omega / \mathrm{m}]$ & $Z_{y}^{e f f}[\mathrm{M} \Omega / \mathrm{m}]$ & $\mathrm{d} Q_{x} / \mathrm{d} N_{b}\left(\cdot 10^{-11}\right)$ & $\mathrm{d} Q_{y} / \mathrm{d} N_{b}\left(\cdot 10^{-11}\right)$ \\
\hline $01-05-2013 \# 1$ & $(2.47 \pm 0.30)$ & $(3.22 \pm 0.27)$ & $(-2.32 \pm 0.29) \cdot 10^{-4}$ & $(-2.92 \pm 0.25) \cdot 10^{-4}$ \\
$01-05-2013 \# 2$ & $(3.87 \pm 0.85)$ & $(3.14 \pm 0.62)$ & $(-3.64 \pm 0.80) \cdot 10^{-4}$ & $(-2.85 \pm 0.56) \cdot 10^{-4}$ \\
\hline Average & $(3.17 \pm 1.15)$ & $(3.18 \pm 0.89)$ & $(-2.98 \pm 1.09) \cdot 10^{-4}$ & $(-5.77 \pm 0.81) \cdot 10^{-4}$ \\
\hline
\end{tabular}

Table 4: Tune slope and effective impedances measured in RHIC Yellow ring.

to the higher beta function. In critical areas, close to the interaction points, BPMs record both plane (i.e. two pairs of striplines). The system is able to record up to 1024 turns. During operation a noise of $\sigma_{n} \simeq 10 \mu \mathrm{m} \mathrm{rms} \mathrm{was} \mathrm{measured} \mathrm{in} \mathrm{the} \mathrm{turn} \mathrm{by} \mathrm{turn} \mathrm{data.}$

From the measured tune shift with intensity we measured a systematically higher impedance for the Blue ring with respect to the Yellow one, even if not in a reproducible way. We tried therefore to apply the impedance localization method in order to get an estimate of the main impedance sources in the Blue ring. The main Blue ring machine parameters used in the measurement are listed in App. B

The data recorded during 15-05-2013 were analyzed with SUSSIX and the integrated phase advance calculated starting from the STAR interaction point. At different intensities the phase advance was expected to shift linearly (analogously with the tune) as can be seen in Fig.7a. The measured data presented an average noise level $N S R \simeq 5 \%$. Using Eq. (7) we can estimate the accuracy for the phase advance slope $\Delta \mu / \Delta N_{b}$ measurement and compare with the expected one.

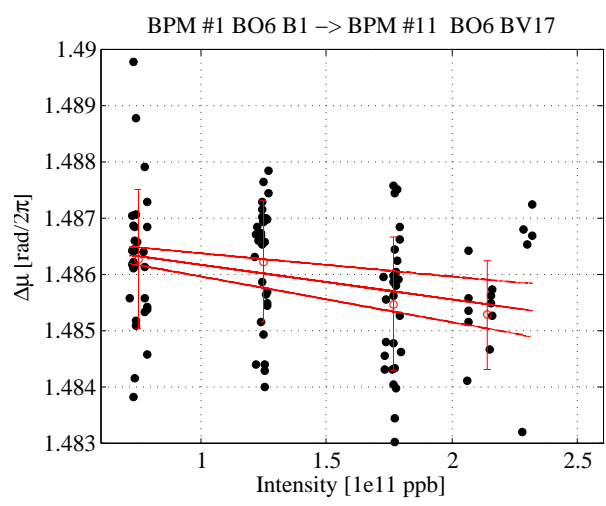

(a)

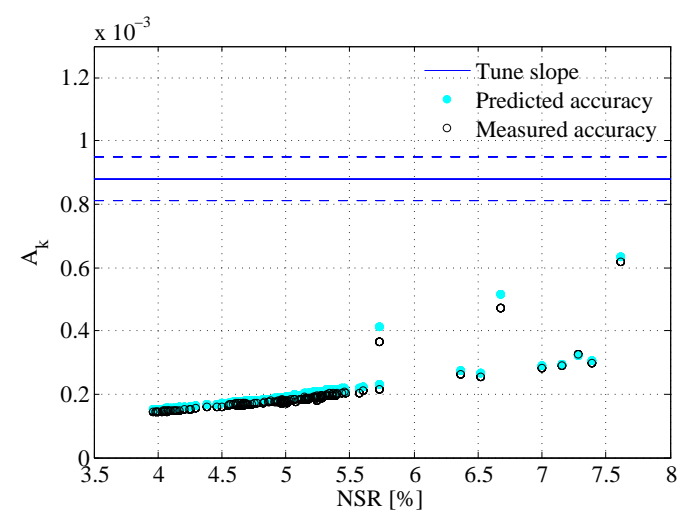

(b)

Figure 7: (a) example of phase advance slope with intensity measured in Blue; (b) comparison of the measured and predicted accuracy with the NSR level for all the measured phase advances (dots) and comparison with the tune slope (thick line) and its accuracy (dashed lines).

Figure $7 \mathrm{~b}$ shows the measured and predicted accuracy compared with the tune shift with intensity (i.e. the total integrated phase advance slope with intensity). A high margin is present and suggests that an impedance of the order of $20 \%$ the total machine impedance could be localized.

Extrapolating the phase advance at 0 -current $N_{b}=0 \mathrm{ppb}$, we can study the machine optics and quantify the accuracy of the MAD-X model used for its description. Figure 8 shows the comparison between model and measurement as well as their relative ratio. The model is in agreement within $30 \%$ to the measured one: this is a limitation for the impedance reconstruction algorithm, where the interpolation over a reliable (usually less than 5\%) model is required in order to localize the most probable impedance sources.

The measured phase advance slope is shown in Fig. 9. The reconstruction algorithm is based on a least-square procedure: defined the set of observers $B$ (i.e. the BPMs) and a set of reconstructors 

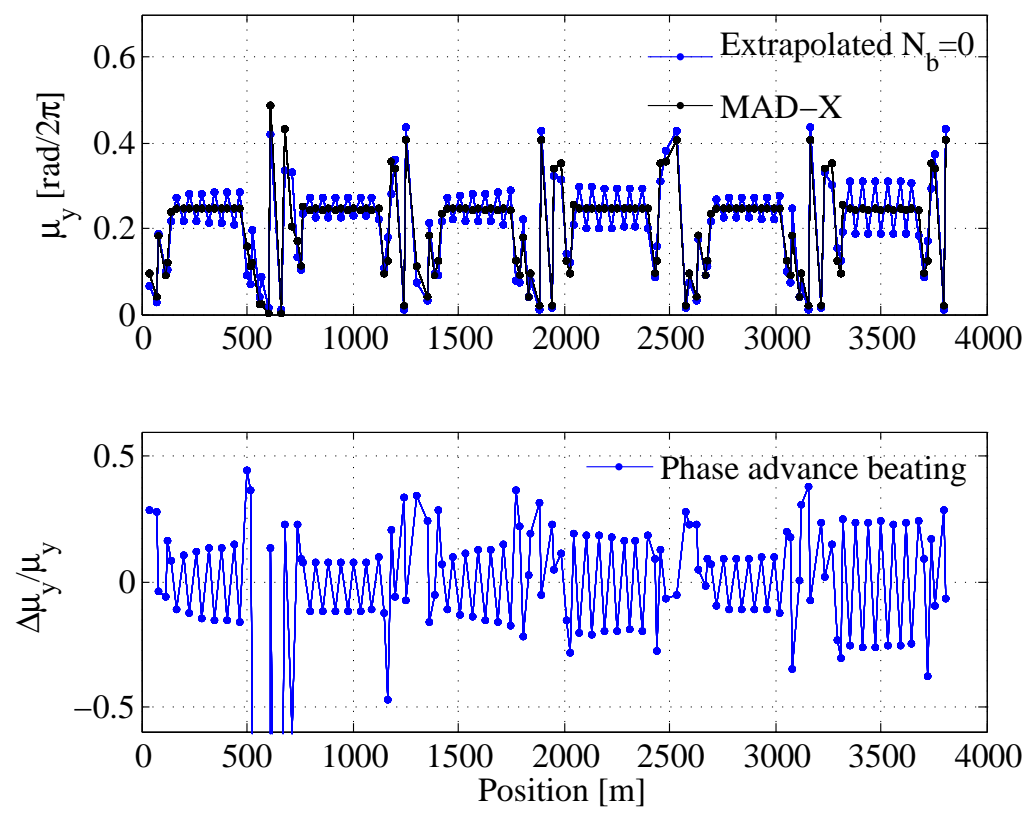

Figure 8: Measured phase advance in Blue compared with the MAD-X model (top) and relative beating (bottom).

$K$ (i.e. the impedance positions), we can calculate the response matrix $S$ at the observers from each of the reconstructors modeled as a thin quadrupole error [5].

The problem can be formulated in finding the best set of reconstructors $K$ in order to match the measured signal at $B$ through $B=S \cdot K$ in a least square sense. In order to reconstruct the possible impedance positions we considered a set of impedance reconstruction points coincident with the BPM position. This choice is imposed by the model accuracy. As it has been shown, the model is accurate only within $30 \%$ : choosing the reconstruction points at the BPM position enables to calculate the response matrix $S$ using the measured phase advances in place of the model ones, being able to calculate a more reliable response matrix.

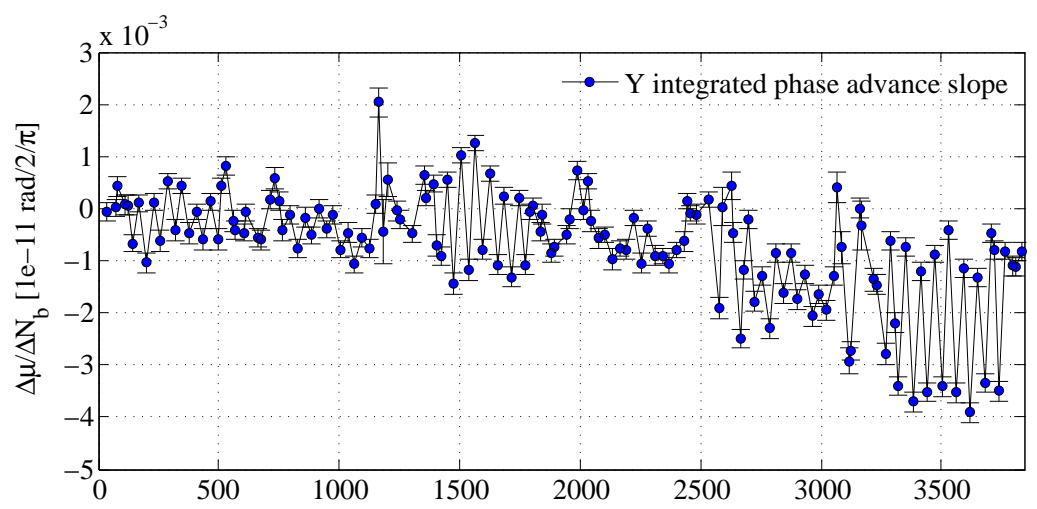

Figure 9: Measured phase advance slope in Blue.

Measured and reconstructed slopes are shown in Fig. 10 as well as the impedance position. While the phase advance $\Delta \mu$ can be measured independently from the model, the $\beta$ function measurement is more involved: if derived from the measured oscillation amplitude it could be affected by the BPM gain calibration, if derived from the phase advance it depends on the model accuracy. Most of the impedance-induced phase advance beating is produced by localized impedance sources around $s \simeq 2500 \mathrm{~m}$ in the machine. This is only a preliminary conclusion due to the limited accuracy of the lattice model of the machine. It is known that part of the complication is due to the difficult modeling of the Snakes, special magnets used to preserve the beam spin direction along 
acceleration. For this reason new measurements are planned without the use of the Snakes and at flat top in order to mitigate the tune drift with time effect.
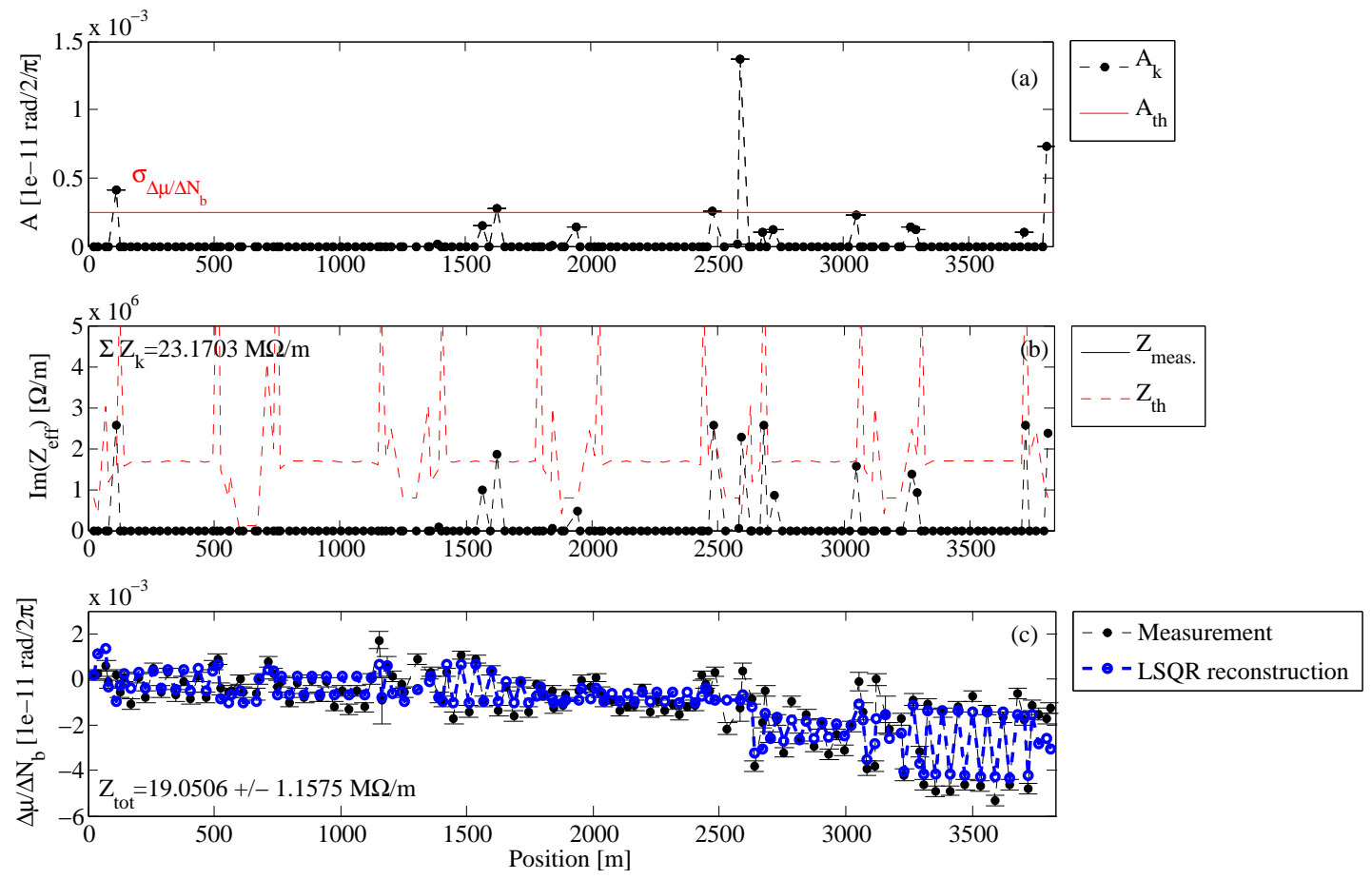

Figure 10: Measured phase advance slope in Blue placing reconstruction kicks at the BPM positions. (a) The reconstructed amplitude of the impedance-induced phase advance beating (in black) with accuracy threshold (in red); (b) The reconstructed impedance locations (in black) with corresponding accuracy threshold (in red); (c) Measured phase advance slope and LSQR reconstruction. 


\section{Conclusions}

The total vertical effective impedance has been evaluated in the AGS machine to be about $Z_{y}^{e f f} \simeq 1.3 \mathrm{M} \Omega / \mathrm{m}$. This estimation has been found to be in good agreement with past longitudinal impedance estimations which suggest the most of the accelerator impedance is due to the resistive wall impedance. Since the longitudinal impedance measurement are not recent, in order to further confirm this result new measurements are expected unless not important machine changes are believed to be occurred.

Measurements in RHIC have been performed on 24-04-2013, 1-05-2013 and 15-05-2013. The impedance of the Blue ring has been found to be significantly higher than the one of Yellow where we have estimated $Z_{x, y}^{e f f} \simeq 3 \mathrm{M} \Omega / \mathrm{m}$ : this discrepancy is not yet understood being the accelerators, in principle, identical. Excluding, for the moment, measurement artifacts we tried to take profit of the good BPM system in order to localize the main Blue impedance sources. Unfortunately, the optics model of the machine turned out to be not enough accurate for the impedance position reconstruction. It is known that part of the complication is due to the difficult modeling of the Snakes, special magnets used to preserve the beam spin direction along acceleration.

From a practical point of view we would suggest to:

1. Repeat the measurement at flat top in order to avoid the parameter drifts that were partially compensated and reduce the impact of the Snakes.

2. Switch off the Snakes in order to get a simpler optic model to refer to.

3. Switch to Ion runs where the optics do not include the Snakes.

4. Perform the localization measurement using the AC dipole excitation to achieve higher data quality and measurement accuracy.

5. Improve the machine optics down-to a $5-10 \%$ in order to improve the localization potentialities.

6. Extend the MD time to 6 hours due to the long and careful chromaticity setting procedure. 


\section{A AGS machine parameters}

These settings were used in the AGS tune shift measurements with protons. This set up refers to the MD of 17-04-2013.

\begin{tabular}{lcr}
\hline \hline & Parameter & Value \\
\hline Circumference & $C$ & $807.1 \mathrm{~m}$ \\
Energy (total) & $E$ & $23.81 \mathrm{GeV}$ \\
Energy (kinetic) & $T$ & $22.87 \mathrm{GeV}$ \\
Gamma & $\gamma$ & 25.38 \\
Gamma transition & $\gamma_{t}$ & 8.5 \\
Revolution period & $T_{o}$ & $2.69 \mu \mathrm{s}$ \\
Vertical Tune & $Q_{y}$ & 8.87 \\
Horizontal Tune & $Q_{x}$ & 8.71 \\
Average $\beta_{x}(s)$ & $\bar{\beta}_{x}$ & $16 \mathrm{~m}$ \\
Average $\beta_{y}(s)$ & $\bar{\beta}_{y}$ & $16 \mathrm{~m}$ \\
Vertical Chromaticity & $\xi_{y}$ & $\simeq 0.16$ \\
Horizontal Chromaticity & $\xi_{x}$ & $\simeq-0.83$ \\
Harmonic number & $h$ & 8 \\
RF frequency & $f_{R F}$ & $2.969 \mathrm{MHz}$ \\
Synchronous phase & $\phi_{s}$ & $\pi$ \\
RF voltage & $V_{R F}$ & $200 \mathrm{kV}$ \\
Synchrotron tune & $Q_{s}$ & $3.6 \cdot 10^{-4}$ \\
\hline
\end{tabular}

Table 5: AGS machine parameters. 


\section{B RHIC}

These settings were used in the RHIC tune shift and impedance localization measurements with protons. This set up refers to the MD of 24-04-2013 and 15-05-2013 in Blue ring and to the MD of 01-05-2013 of Yellow Ring. The two accelerator rings are in principle specular.

\begin{tabular}{lcr}
\hline \hline & Parameter & Value \\
\hline Circumference & $C$ & $3833.8451 \mathrm{~m}$ \\
Energy (total) & $E$ & $25 \mathrm{GeV}$ \\
Energy (kinetic) & $T$ & $24.06 \mathrm{GeV}$ \\
Gamma & $\gamma$ & 26.64 \\
Gamma transition & $\gamma_{t}$ & 23.50 \\
Revolution period & $T_{o}$ & $12.79 \mu \mathrm{s}$ \\
Vertical Tune & $Q_{y}$ & 29.691 \\
Horizontal Tune & $Q_{x}$ & 28.695 \\
Average $\beta_{x}(s)$ & $\bar{\beta}_{x}$ & $\simeq 57.3 \mathrm{~m}$ \\
Average $\beta_{y}(s)$ & $\bar{\beta}_{y}$ & $\simeq 50.2 \mathrm{~m}$ \\
Vertical Chromaticity & $\xi_{y}$ & $\simeq 0.07$ \\
Horizontal Chromaticity & $\xi_{x}$ & $\simeq 0.07$ \\
Harmonic number & $h$ & 2520 \\
RF frequency & $f_{R F}$ & $197 \mathrm{MHz}$ \\
Synchronous phase & $\phi_{s}$ & $\pi$ \\
RF voltage & $V_{R F}$ & $300 \mathrm{kV}$ \\
Synchrotron tune & $Q_{s}$ & $6.47 \cdot 10^{-5}$ \\
\hline
\end{tabular}

Table 6: RHIC machine parameters. 


\section{References}

[1] R. Calaga. Transverse impedance measurements in rhic. 2010. APEX Workshop, Stony Brook.

[2] E. Métral. Uspas2009 course, albuquerque, usa. June.

[3] Brown K. Glenn J.W. Raka E. Ryan J. Blaskiewicz, M. Spill structure in intense beams. 2003. Proc. of PAC 2003.

[4] A. Chao. Physics of Collective Beam Instabilities in High Energy Accelerators. Wiley Interscience publication. Wiley, 1993.

[5] N.Biancacci. Improved techniques of impedance calculation and localization in particle accelerators. 2014. PhD Thesis.

[6] R Bartolini and F Schmidt. SUSSIX: A computer code for frequency analysis of non-linear betatron motion. 1998 .

[7] T.J. Shea and R.L. Witkover. RHIC instrumentation. 1998.

[8] T. Satogata, R. Calaga, P. Cameron, P. Cerniglia, J. Cupolo, A. Curcio, W. C. Dawson, C. Degen, J. Gullotta, J. Mead, R. Michnoff, T. Russo, and R. Sikora. RHIC BPM System Modifications and Performance. 2005. 